University of South Florida

DIGITAL COMMONS

Digital Commons @ University of

@ UNIVERSITY OF SOUTH FLORIDA

South Florida

Nursing Faculty Publications

College of Nursing

2012

\title{
Development of a Communication Intervention to Assist Hospitalized Suddenly Speechless Patients
}

\author{
Carmen S. Rodriguez \\ University of Florida \\ Meredeth A. Rowe \\ University of South Florida, mrowe1@health.usf.edu \\ Brent Koeppel \\ Loris A. Thomas \\ University of Florida \\ Michelle S. Troche \\ University of Florida
}

See next page for additional authors

Follow this and additional works at: https://digitalcommons.usf.edu/nur_facpub

Part of the Nursing Commons

\section{Scholar Commons Citation}

Rodriguez, Carmen S.; Rowe, Meredeth A.; Koeppel, Brent; Thomas, Loris A.; Troche, Michelle S.; and Paguio, Glenna, "Development of a Communication Intervention to Assist Hospitalized Suddenly Speechless Patients" (2012). Nursing Faculty Publications. 15.

https://digitalcommons.usf.edu/nur_facpub/15

This Article is brought to you for free and open access by the College of Nursing at Digital Commons @ University of South Florida. It has been accepted for inclusion in Nursing Faculty Publications by an authorized administrator of Digital Commons @ University of South Florida. For more information, please contact digitalcommons@usf.edu. 


\section{Authors}

Carmen S. Rodriguez, Meredeth A. Rowe, Brent Koeppel, Loris A. Thomas, Michelle S. Troche, and Glenna Paguio 
Technol Health Care. 2012 ; 20(6): 489-500. doi:10.3233/THC-2012-0695.

\title{
Development of a communication intervention to assist hospitalized suddenly speechless patients
}

\author{
Carmen S. Rodriguez ${ }^{a,{ }^{*}}$, Meredeth Rowe ${ }^{b}$, Brent Koeppel ${ }^{\mathrm{c}}$, Loris Thomas ${ }^{\mathrm{d}}$, Michelle S. \\ Troche $^{e}$, and Glenna Paguio ${ }^{f}$ \\ aAdult and Elderly Department, College of Nursing, University of Florida, Gainesville, FL, USA \\ ${ }^{\text {b} C o l l e g e ~ o f ~ N u r s i n g, ~ U n i v e r s i t y ~ o f ~ S o u t h ~ F l o r i d a, ~ T a m p a, ~ F L, ~ U S A ~}$ \\ ${ }^{\circ}$ Chameleon Adaptiveware, LLC, Natick, MA, USA \\ dUniversity of Florida, Gainesville, FL, USA \\ eDepartment of Speech, Language, and Hearing Sciences, Division of Speech Pathology, \\ University of Florida, Gainesville, FL, USA \\ fUniversity of Florida, Clinical Research Center, Shands at The University of Florida, Gainesville, \\ FL, USA
}

\section{Abstract}

\begin{abstract}
Healthcare staff face significant challenges while caring for hospitalized patients experiencing sudden inability to verbalize their needs (sudden speechlessness). Familiar methods of communication such as non-verbal strategies are limited and often fail to assist suddenly speechless patients (SS) communicate their needs. Consequently, strategies tailored to the needs of hospitalized speechless patients are necessary, and must consider factors intrinsic to the patients and the complexities of the acute care environment. The feasibility and usability of a multifunctional prototype communication system (speech-generating device) tailored to the needs of hospitalized SS patients was evaluated in this pilot study. Adult SS patients admitted to the intensive ewe setting ( $n=11)$ demonstrated independent use of a multi-functional communication system that integrated messages and strategies tailored to the needs of the hospitalized SS patient. Participants reported high satisfaction levels and considered the use of the technology of high importance during an SS event. Additional research should focus on evaluating the impact of technology specific communication interventions on enhancing the communication process between SS patients and healthcare staff.
\end{abstract}

\section{Keywords}

Communication intervention; sudden speechlessness; acute care setting

(C) 2012 - IOS Press and the authors. All rights reserved

*Corresponding author: Carmen S. Rodriguez, Adult and Elderly Department, University of Florida-College of Nursing, 101 South Newel Drive, Gainesville, FL 32610, USA. Tel.: +1 352273 6328; rodrics@ufl.edu. 


\section{Introduction}

Healthcare staff often face the challenge of caring for patients experiencing sudden inability to verbalize their needs (sudden speechlessness). Suddenly speechless (SS) patients, who are often recovering from surgery/trauma to organs that are critical to speech, or patients who are undergoing respiratory intubation, are challenged by communication constraints that leave them unable to communicate with familiar methods, and/or the absence of a new mechanism with which they can communicate their needs. Current non-verbal strategies that facilitate SS patients' communication abilities are time-consuming and inadequate as they fail to improve communication between SS patients and nursing staff. Strategies that address the communication needs of SS patients must consider factors intrinsic to the patients as well as the viability of implementing the strategy in a complex health care environment. The primary purpose of this pilot study was to test the feasibility and usability of a multifunctional prototype communication system that is tailored to the needs of hospitalized SS patients. The following research questions guided the study:

1. Can suddenly speechless patients who are admitted to the critical care setting independently activate strategies that are integrated in the communication system on command (i.e. direct selection pictorial hot-buttons, handwriting, typing), to communicate safety, care and comfort needs?

2. What are the difficulties encountered by hospitalized SS patients using the communication system?

3. How do study participants rate the level of importance and satisfaction with the use of the communication system?

\section{Background}

In the hospital setting, SS patients are often found in intensive care units, intermediate care units or medical surgical units after undergoing respiratory intubation, placement of a tracheotomy, or while recovering from head and neck surgery or trauma. Despite where SS patients are admitted - traditional medicine units or critical care units - or the rationale for developing speechlessness, the absence of reliable methods to communicate is associated with negative emotional patient outcomes such as anger [1], fear [2], and frustration [3-5]. The challenge of assisting SS patients' communication in an environment with limited and often ineffective resources is shared by healthcare professionals working directly with SS patients (e.g., doctors, nurses, speech-language pathologists).

Speech-Language Pathologists (SLPs) presently aim to identify methods that enhance the SS patient's ability to communicate wants and needs with healthcare professionals and caregivers. However, currently there are few sophisticated tools available to SLPs for the management of transiently speechless patients who, although they have sufficient cognitive resources to support communication, cannot verbally communicate due to a deficit in structure or function of speech mechanisms. In the case of intubation, tracheotomy, or recent laryngectomy, an electro larynx can be given to patients to facilitate communication, but because functional use of an electro larynx is limited to persons without oral intubation, swelling/pain in the neck region, or reduced range of motion of articulators, few transiently 
speechless patients meet these criteria. Additionally, patients with minimal range of motion or strength of upper extremities may require a caretaker to place the electro larynx to their neck, and this limits the patient's ability to independently communicate.

In order to facilitate communication with patients, basic communication pictures and alphabet boards are commonly used by SLPs and other healthcare professionals. Picture boards are usually comprised of randomly selected pictures that are thought or assumed to be applicable to speechless patients in the medical setting. However, alphabet boards are tedious to use and because they limit the ability to produce novel utterances or expeditiously express emergent needs, often result in inefficient communication and poor patient compliance. Furthermore, the use of picture boards have not been validated and often include pictures that are not useful to patients and their communication needs. While these boards may help patients express their most basic needs, they often do not allow patients to express wants that are essential to maintaining quality of life.

Working with SS patients is challenging for nurses because the transmission of messages with nonverbal expressions can be difficult to interpret, and communication strategies for SS patients may vary within a setting, forcing nurses to seek reliable alternatives in the midst of a demanding healthcare situation. While SS patients commonly write their needs on a pad, or attempt to create words (one letter at a time) using an alphabet board, in the immediate aftermath of a critical health event (e.g., cardiac or head and neck surgery, need for respiratory intubation), or when fatigue prevails, these methods are often inadequate and time consuming. Patients' limitations coupled with undependable approaches substantiate the need to identify effective strategies that can enhance communication between nurses and SS patients.

\section{Communication intervention for SS patients}

The development of reliable approaches is essential to facilitate self-expression in SS patients, and to decrease frustration and dissatisfaction associated with care received [4-9]. Speech generating devices (SGDs), which have been consistently used by individuals with permanent speech impairments (e.g., neuromuscular diseases, brain injuries) in the home or school setting, have also proven to be an acceptable communication approach in a limited number of pilot studies with hospitalized SS patients [6,10-12], However, because SGDs have the potential to solve communication problems that are experienced by hospitalized SS patients, considering requirements intrinsic to the SS population as well as practicalities associated with the acute care environment is necessary before these communication devices can successfully be incorporated into a hospital setting.

\subsection{Intrinsic requirements}

Because SS often develops without warning, there is no opportunity for the implementation of systematic teaching strategies prior to its occurrence. Most patients that require an unexpected airway intubation or emergency surgery that results in SS become acquainted of their inability to verbalize their needs ex post facto. For a limited number of patients (e.g., patients scheduled for cardiac or head and neck surgery), awareness of the potential to lose their voice may occur during the pre-operative period while information about potential 
procedures, complications, and informed consent is explained. As a result, the following characteristics were considered during the developmental and refinement stages of the SGD prototype that was evaluated in this pilot study: 1) How simple was facilitating implementation of a communication intervention involving the use of technology at the patient's bedside? 2) Would health care staff and SS patients find the communication intervention easy to learn how to use? 3) Was the technology been effective when timely assistance was needed? and 4) Would the intervention appropriately meet the communication needs of SS patients as recovery unfolds [12]. This SGD consisted of software that facilitated the use of multifunctional strategies to communicate (e.g., direct selection of pre-recorded messages associated with symbols, writing and typing), hardware components including a tablet computer, and an urgent button. The urgent button, which consisted of a self-contained device that announced "I need help", was provided to each participant so that they could summon help in the event of an emergency, such as prototype system failing.

\subsection{Acute care environment}

Prior to initiating this study, consultation was coordinated with the Infection Control, Safety and Clinical Engineering Departments and the following requirements were implemented: the SGD and tablet computer were disinfected after use by each participant, screen protectors were used and discarded after each participant completed the study, and extension cords were not allowed. Additionally, the SGD was mounted in a mobile unit to facilitate access and use in areas with limited space.

\section{Conceptual framework}

The Process of Interpretation in Response to Voicelessness Framework [7] was adapted to provide conceptual basis to this study. According to Happ, voicelessness represents communication barriers that limit patients' abilities to convey thoughts, feelings, desires and needs to others, resulting in delays to communicate symptoms or problems, misinterpretation of non-verbal communication, and feelings of fear and anxiety. According to Happ, integrating communication interventions at a time when critical information must be shared between clinicians and patients may result in improvement of ease, quality, frequency, and success of the communication process.

This pilot study evaluated the feasibility of integrating a communication intervention for hospitalized SS patients. Integrating a communication intervention that is tailored to the needs of SS patients was considered critical in order to decrease difficulties associated with an inability to verbalize needs and to enhance the communication process during the hospital stay.

\section{Methods}

The use of a programmable SGD, which uses software incorporated in a tablet computer, as a communication intervention for hospitalized patients was evaluated over participants' hospital stay (maximum of 10 days). Approval from the researchers' Institutional Review Board was obtained before the study was implemented in three adult critical care units 
(surgical, medical, and cardiac) as well as a medical surgical unit where study participants were transferred (until completion of study) at a tertiary care institution in the southeast region of the United States.

\subsection{Sample}

The study inclusion criteria were: 1 ) $\geq 21$ years of age; 2) currently experiencing SS (H \& N surgical in-patients 2-10 days post-surgery) or hospitalized intubated patients who were being weaned from the ventilator; 3) no delirium as measured by the Confusion Assessment Method Shortened Version scale; 4) score $< \pm 2$ in the Richmond-Agitation-Sedation scale; and 5) able to read/speak English. Participants unable to use at least one arm or with longterm speechlessness resulting in the use of an adaptive speech generating device (SGD) were excluded.

\subsection{Recruitment and consent}

$\mathrm{H} \& \mathrm{~N}$ surgical patients were identified during their pre-operative clinic appointment and given the option to immediately sign the consent form or sign it upon hospital admission. Patients who were undergoing respiratory intubation were identified during their hospital stay after the ventilator weaning process had been initiated. All subjects volunteered to participate in the study and signed a consent form.

\subsection{Data collection}

Data collection was conducted on the admitting units as well as on medical-surgical units where participants were transferred when critical care management was no longer needed. The following data were collected during the study: demographics, presence of delirium and agitation/sedation, usability of communication intervention, and satisfaction/importance of using communication intervention. The study measures and timeline for implementation of study are outlined in Table 1.

\subsection{Procedure}

After research staff determined that participants were not experiencing delirium and had a RASS score $< \pm 2$, each participant received an SGD and an orientation on how to use the device. The Usability of Communication Intervention Form was completed by research staff every day of the study until the patient was discharged. Participants were given refresher information as needed regarding the use of the SGD and urgent button. Prior to discharge, participants completed the Patient Satisfaction and Usability Instrument. Assistance was provided by the research staff (writing participants' feedback and rating of each item) as requested. To evaluate the functionality of the SGD and urgent button, research staff tested the technology on every data collection day. The unit clerk's understanding of a message generated by the SGD via the hospital call system was assessed daily for participants who were transferred to a medical surgical unit. 


\subsection{Data analysis}

Descriptive statistics were used to summarize the sample demographics, clinical variables, and scores for all satisfaction and importance items. The Kappa statistic was used to test for reliability of The Patient Satisfaction and Usability Instrument.

\section{Results}

\subsection{Participant characteristics}

A total of twenty four subjects consented to participate in the study. Three subjects (12.5\%) completed only one day of the study due to declining health. Seven consented subjects did not participate in the study due to the following: discharge to Rehabilitation Hospital ( $n=1$; $14.3 \%)$; declining health status $(n=1 ; 14.3 \%)$; effective use of Passy Muir speaking valve ( $n=1 ; 14.3 \%)$; presence of delirium $(n=1 ; 14.3 \%)$; surgery cancellation $(n=1 ; 14.3 \%)$; and no interest in participation $(n=2 ; 28.6 \%)$. The final sample consisted of 11 participants with an age range of $40-72$ years $(=13.27 \pm 2.90)$. Participants were mostly male $(81.8 \%)$, White (90.9\%), with more than 8 years of education (91\%), who became SS as a result of airway intubation (100\%). Approximately $73 \%$ of the participants had head and neck cancer and were recovering from a surgical intervention that resulted in $\operatorname{SS}\{\operatorname{laryngectomy}(n=5)$; floor of mouth ( $n=1)$; base of tongue $(n=1)$; skin cancer with extensive involvement and oral-facial defect $(n=1)\}$. The average number of days in the study was 6 days (range 1-10; $\mathrm{SD}=2.75)$. Table 2 describes the characteristics of the participants.

The CAM and the RAS were used as pre-screening tools prior to consenting potential participants and/or daily data collection. Initial RAS and CAM evaluations that were conducted prior to providing SGDs to participants demonstrated that $91 \%$ of participants did not experience behaviors that were consistent with delirium, restlessness or sedation. Because one participant (9\%) demonstrated light sedation (RAS $=-2)$ and acute changes in mental status upon initial evaluation, a demonstration about how to use the SGD was not provided until symptoms resolved, usually over a period of 24 hours. Another participant demonstrated acute changes in mental status while the study was in progress (day 6), but appropriately recovered and was able to complete the study.

\subsection{SS patients' ability to activate strategies integrated in the communication system on command}

6.2.1. Hot-buttons-Participants demonstrated the ability to independently use the SGD from day 1 until completion of the study. At a minimum, seventy-three percent of participants (range, $72.7 \%-100 \%$ ) were able to independently activate five hot-buttons on command on day 1 (Fig. 1), after a 10 minute review about how to use the SGD. Consistent independent performance was observed throughout the study (Table 3). One participant was unable to physically push one out of five hot-buttons at two different points of data collection, requiring significant assistant from research staff. Additionally, for a small number of participants $(n=3)$, the evaluation of their ability to activate messages on command was not possible for a range of 1-2 data collection points due to the following reasons: the unit was off for the procedure and/or transfer $(n=1)$ and the participant experienced drowsiness $(n=1)$ or fatigue $(n=1)$. 
6.2.2. Writing and typing functions-From a total of 52 data collection points, the handwriting screen was accessed independently $92.3 \%$ of the times $(n=48)$. The typing screen was accessed independently $94.4 \%$ of the times ( $n=34$ of 36 data collection points). Minimal assistance was needed to activate the typing function during day 2 by one participant. Minimal assistance to activate the handwriting function was required by two participants on day 1 and one participant on day 5 .

6.2.3. Use of the urgent button-From a total of 63 data collection points, the Urgent Button (UB) was independently activated $83 \%$ of the times by study participants. The most common difficulty experienced by participants $(n=3)$ related to temporary inability to locate the UB for activation. On Day 1 of the study two participants (18\%) required minimal assistance (less than two prompts) to activate the UB and one participant required considerable assistance (more than 2 prompts) to locate the UB for activation. One participant (9.0\%) activated the Emergency hot-button instead of the UB. A review about where the UB was located and procedure to activate the UB was provided every time participants required minimal or considerable assistance to activate the device. One participant required assistance locating the UB for 3 consecutive days with independent activation reported for the rest of time the participant was in the study. Another participant required considerable assistance locating the UB throughout the study, on days 2, 3, 5 and 9.

\subsubsection{Difficulties encountered by participants when use of SGD was} requested-Several participants encountered the following difficulties when use of the SGD was requested: they were unable to physically push requested icon $(n=2 ; 18 \%)$; their eyeglasses were not available, limiting visual acuity $(n=2)$; the level of sedation impaired the correct use of device ( $n=1 ; 9 \%)$; and their writing was illegible $(n=2 ; 18 \%)$. Two participants were unable to identify messages independently upon request and two participants experienced difficulty using a stylus to activate the correct hot-buttons. Difficulty activating the hot-buttons was associated with having a pulse oximeter on the dominant hand $(n=1 ; 9 \%)$, and/or wrist restraints $(n=1 ; 9 \%)$, and resulted in the activation of the hot-button next to the one that was requested by research staff.

Re-teaching of device use was necessary for three participants (27\%). The need for a review was associated with difficulty to activate three hot buttons on command $(n=1 ; 33 \%)$, difficulty to locate the urgent button $(n=2 ; 66 \%)$, or the need to reinforce information as a result of acute mental status changes $(n=1 ; 33 \%)$.

\section{Effectiveness of communication system outside of the critical care setting}

\subsection{Clerk's understanding of message via unit call button}

Participants transferred to medical surgical units where the hospital call system was in use ( $n$ $=10$ ) activated the system at forty different data collection points, and upon response from the clerk, proceeded to activate a message from the device. Ninety five percent $(n=38)$ of the messages generated by participants via the hospital call system were understood by the clerks. 


\subsection{Accessibility of the communication system}

During the study, the SGD was accessible in the participants' rooms approximately ninetysix percent of the times $(n=66)$ data were collected. The SGD was accessible to the participants within arm's reach $52 \%$ of the times $(n=34)$. For approximately $44 \%$ of the times $(n=29)$, the device was in the room but beyond the participants' reach. The rationale for not having the SGD within arm's reach of participants included the following: the device was moved by the nurse, physical therapy or relative and not returned within the participant's reach $(n=12)$; unknown rationale $(n=11)$; the device was moved by the study participant (e.g. ambulatory participant) and relocated as needed $(n=4)$; the participant requested the device be moved $(n=2)$. During the study, the device was not found in the room of two participants who were transferred from the intensive care unit to a medical surgical floor (3\%) at one data collection point (e.g., day of transfer). Additionally, one participant's device was stored at the nurses' station after patient extubation and recovery of speech within 24 hours of initiation of the study (1.5\%).

\subsection{Usability of communication system}

Usability of the SGD was evaluated by determining if the device was functional at 66 data collection points. The device was on and functional at capacity $68 \%$ of the times $(n=45)$. The SGD was found non-functional (powered off) for the following reasons: it was found unplugged and powered off for unknown reasons $(n=10 ; 15.1 \%)$; the device was not set-up after it was transferred to another unit or room (or procedure $n=3 ; 4.54 \%$ ); participants accidentally pulled the device from the dock, resulting in automatic shut-off $(n=1 ; 1.51 \%)$; and the device was moved in the room and then left unplugged $(n=2 ; 3 \%)$. Additionally, the SGD's did not produce speech $(n=2 ; 3 \%)$, hot-buttons froze $(n=2 ; 3 \%)$, and/or both speech and hot-buttons were non-functional $(n=1 ; 1.5 \%)$ due to unknown causes. A re-boot of the communication system made devices operational within the same data collection visit each time usability issues were identified.

\section{Level of importance and satisfaction with the use of the communication system}

\subsection{Importance and satisfaction}

Participants rated their importance and satisfaction level by using The Patient Satisfaction and Usability Instrument ( 1 = strongly agree, 2 = agree, 3 = neither agree or disagree, $4=$ disagree, 5 = strongly disagree). Six items measured satisfaction based on use of device functions, ease of device use, and ability to report symptoms and/or improve communication with nursing staff. The mean score for all satisfaction items was $1.5(n=11 ; \mathrm{SD}=0.29$; range $=1.16-2.0$ ), indicating that participants were satisfied with use of the SGD during their hospital stay. One participant strongly disagreed that the prototype device was easy to use, based on the size ("too big, in the way".

Four items measured importance specific to availability of the SGD for use by SS patients and importance of device functions. The mean score for all importance items was $1.61(n=$ $11 ; \mathrm{SD}=0.55$; range 1-3.00), indicating that participants considered the use of the SGD and its functions of importance while experiencing SS. One participant strongly disagreed that 
having picture hot-buttons was an important function, indicating his/her preference to "write fast", providing a better rating to writing as a function included on the device.

Single item reliability was determined for importance in having the SGD available for use, and ease of use of the SGD. Results associated with importance of having the SGD indicated a Kappa $=0.421,95 \%$ CI $(-0.243,1.00)$, and a Kappa $=0.388,95 \%$ CI $(-0.011,0.789)$ for ease of use of the SGD. The inclusion of a small sample size limits ability to generate a precise reliability coefficient and significance of findings.

\section{Discussion}

The primary purpose of this pilot study was to test the feasibility and usability of a multifunctional communication system with hospitalized SS patients. In this pilot study, adult SS patients who were admitted to an intensive care unit demonstrated independent use of a communication system that integrated messages and strategies tailored to the needs of the hospitalized SS patient. This communication system facilitated the communication of safety, care and comfort needs experienced by SS patients as recovery evolved, and until discharge from the institution. Adaptations for use of the nurse call system, a component of the standard of care in the acute care setting, were successful in facilitating participants' communication of needs as they were transferred to a medical surgical unit. Participants were strongly satisfied with the use of the communication system and considered the strategies that were included in the communication system important while they were hospitalized and experiencing SS.

Study participants were cognitively functional over the majority of the time they were enrolled in the study, and they represented various educational levels $\left(8^{\text {th }}\right.$ grade to professional degrees) as well as adult age groups. Consistent with literature findings, barriers such as the need to adapt to a challenging acute care environment and limited familiarity with the communication system did not limit participants' ability to use the device [6, 10-12]. In fact, over 70\% percent of the participants $(n=8)$ did not require reteaching of device use during their hospital stay. This area should be explored further as including participants with an educational level beyond the eighth grade limits the ability to generalize findings to individuals with less education and/or lower literacy levels.

Difficulties that were encountered by study participants were consistent with those documented in the literature, including neurocognitive factors such as the presence of delirium, and issues with coordination of the upper extremities [18]. Study findings suggest that the protocol established to assess for the presence of cognitive status prior to consenting participants, as well as daily pre-screening prior to data collection, was effective in identifying participants with acute cognitive changes. Likewise, daily evaluation of participants' use of the device and technology functions helped to identify participants who exhibited occasional difficulty with the device or accessories (i.e. stylus). Consistent reassessments of study participants' skills and device functionality must be considered when conducting studies that explore the use of technology-based communication interventions for SS patients. Future studies need to examine alternate methods that provide SS patients with consistent accessibility to communication devices. 
During studies that involve exploring the use of SGDs in the acute care setting, the frequent removal of the SGD after daily care or placement of the SGD at a difficult-to-reach location represented a challenge $[10,12]$. When the SGD was mounted on a mobile unit, participants could find it more easily, but maintaining the device at a reachable distance and functional setting (plugged in/charged) was still difficult. Strategies to provide consistent accessibility of communication devices to the SS patients must be considered, in particular, attaching the device to an area closer to the patient rather than to a mobile unit. Thus, in the future, expanding patient and health care educational sessions to relatives will help increase understanding regarding the importance of keeping the device within reach for SS patients.

Most participants required minimal instruction to activate the urgent button (UB), which is an independently functioning call button that announced the need for assistance to nursing staff, who could reset it once they attended to the patient. Anecdotal findings shared by participants validated the role of the communication system (including the urgent button) to summon help during emergency situations via the nurse call system. However, further testing of the UB and the SGD will assist in determining the potential benefit and usefulness of each device in helping hospitalized SS patients communicate their needs.

The inclusion of a larger sample of SS patients will also assist in overcoming study limitations such as the selection of a convenience sample and generalization of findings. Future research efforts should incorporate a comparison between a control and intervention group to evaluate if integrating the communication intervention enhances the SS patient's ability to communicate with healthcare staff. Testing the intervention beyond the realms of the critical care setting will facilitate the evaluation of the intervention with other SS populations.

\section{Application}

Successful use of the communication system by acutely ill participants supports the adaptation of strategies to enhance the communication process for SS patients despite the complexity associated with an admission to the critical care setting and limited time to learn the system. Continuing the development and refinement of the communication intervention is consistent with the goal of improving the standard of care for SS patients. The provision of adequate and reliable communication strategies at the bedside is instrumental to enhance the communication process between SS patients and nurses as well as other healthcare staff. Moreover, further development of this technology also has the potential to benefit other groups of patients with the potential to experience SS, including individuals experiencing SS who are not proficient in English.

The active involvement of nurses in the development of interventions for SS patients is a priority because, as the bedside providers of care, they have a unique perspective that is essential to accomplishing the following: improving the quality of care for SS patients by tailoring strategies to their specific needs as well as informing changes that will profoundly impact clinical practice. 


\section{Acknowledgments}

Funded by: NINR SBIR Grant \# 1R42NR010842-01*.

This work was supported in part by NIH awards UL1RR029890, KL2RR029888.

\section{References}

1. Wunderlich RJ, Perry A, Lavin MA, Katz B. Patients' perceptions of uncertainty and stress During weaning from mechanical ventilation. Dimens Crit Care Nurs. 1999 Jan-Feb;18(1):8-12. [PubMed: 10639993]

2. Menzel LK. Factors related to the emotional responses of intubated patients to being unable to speak. Heart Lung. 1998 Jul-Aug;27(4):245-252. [PubMed: 9713716]

3. Patak L, Gawlinski A, Fung NI, Doering L, Berg J. Patients' reports of health care practitioner interventions that are related to communication during mechanical ventilation. Heart Lung. 2004 Sep-Oct;33(5):308-320. [PubMed: 15454910]

4. Rodriguez, CS. Pain measurement in elderly head and neck cancer patients with communication impairments. Tampa, FL: University of South Florida; 2003.

5. Rodriguez CS, VanCott ML. Speech impairment in the post-operative head and neck cancerpatient: Nurses and patients perceptions. Qualitative Health Research. 2005; 15(7):897-911. [PubMed: 16093369]

6. Costello JM. AAC intervention in the intensive care unit: The Children's Hospital Boston model. Augment Altern Commun. 2000; 16(3):137-153.

7. Happ MB. Interpretation of nonvocal communication and the meaning of voicelessness in critical care. Soc Sci Med. 2000; 50:1247-1255. [PubMed: 10728845]

8. Stovsky B, Rudy E, Dragonette P. Comparison of two types of communication methods used After cardiac surgery with patients with endotracheal tubes. Heart Lung. 1988 May; 17(3):281-289. [PubMed: 2966780]

9. Ashworth PM. Staff-patient communication in coronary care units. J Adv Nurs. 1984 Jan; 9(1):3542. [PubMed: 6561215]

10. Happ MB, Roesch TK, Garrett K. Electronic voice output communication aids for temporarily nonspeaking patients in a medical intensive care unit. Heart and Lung. 2004; 33(2):92-101. [PubMed: 15024374]

11. Happ MB, Roesch TK, Kagan SH. Patient communication following head and neck cancer surgery: A pilot study using electronic speech-generating devices. Oncol Nurs Forum. 2005 Nov; 32(6):1179-1187. [PubMed: 16270113]

12. Rodriguez C, Rowe M. Use of a speech-generating device for hospitalized postoperative patients with head and neck cancer experiencing speechlessness. Oncology Nursing Forum. 2010; 37(2): 199-205. [PubMed: 20189925]

13. Miglietta MA, Bochicchio G, Scalea TM. Computer-assisted communication for critically ill patients: A pilot study. J Trauma. 2004 Sep; 57(3):488-493. [PubMed: 15454792]

14. Ely EW, Truman B, Shintani A, Thomason JW, Wheeler AP, Gordon S, Francis J, Speroff T, et al. Monitoring sedation status over time in ICU patients: Reliability and validity of the Richmond Agitation-Sedation Scale (RASS). Jama. 2003 Jun 11; 289(22):2983-2991. [PubMed: 12799407]

15. Sessler CN, Gosnell MS, Grap MJ, Brophy GM, O’Neal PV, Keane KA, et al. The Richmond Agitation-Sedation Scale: Validity and Reliability in Adult Intensive Care Unit Patients. Am. J. Respir. Crit. Care Med. 2002 Nov 15; 166(10):1338-1344. PMID:12421743. [PubMed: 12421743]

16. Demers L, Monette M, Lapierre Y, Arnold DL, Wolfson C. Reliability, validity, and applicability of The Quebec User Evaluation of Satisfaction with assistive Technology (QUEST 2.0) for adults with multiple sclerosis. Disabil Rehabil. 2002; 24(1-3):21-30. [PubMed: 11827151]

17. Demers L, Wessels R, Weiss-Lambrow R, Ska B, Witte L. An international content validation of The Quebec User Evaluation of Satisfaction with assistive technology (QUEST). Occup Ther Int. 1999; 6(3):159-175. 
18. Garrett, KL.; Happ, MB.; Costello, J.; Fried-Oken, MB. AAC in the Intensive Care Unit. In: Beukelman, DR.; Garrett, KL.; Yorkston, KN., editors. Augmentative Communication Strategies for Adults with Acute and Chronic Medical Conditions. Paul H. Brookes Publishing Co.; 2007. 


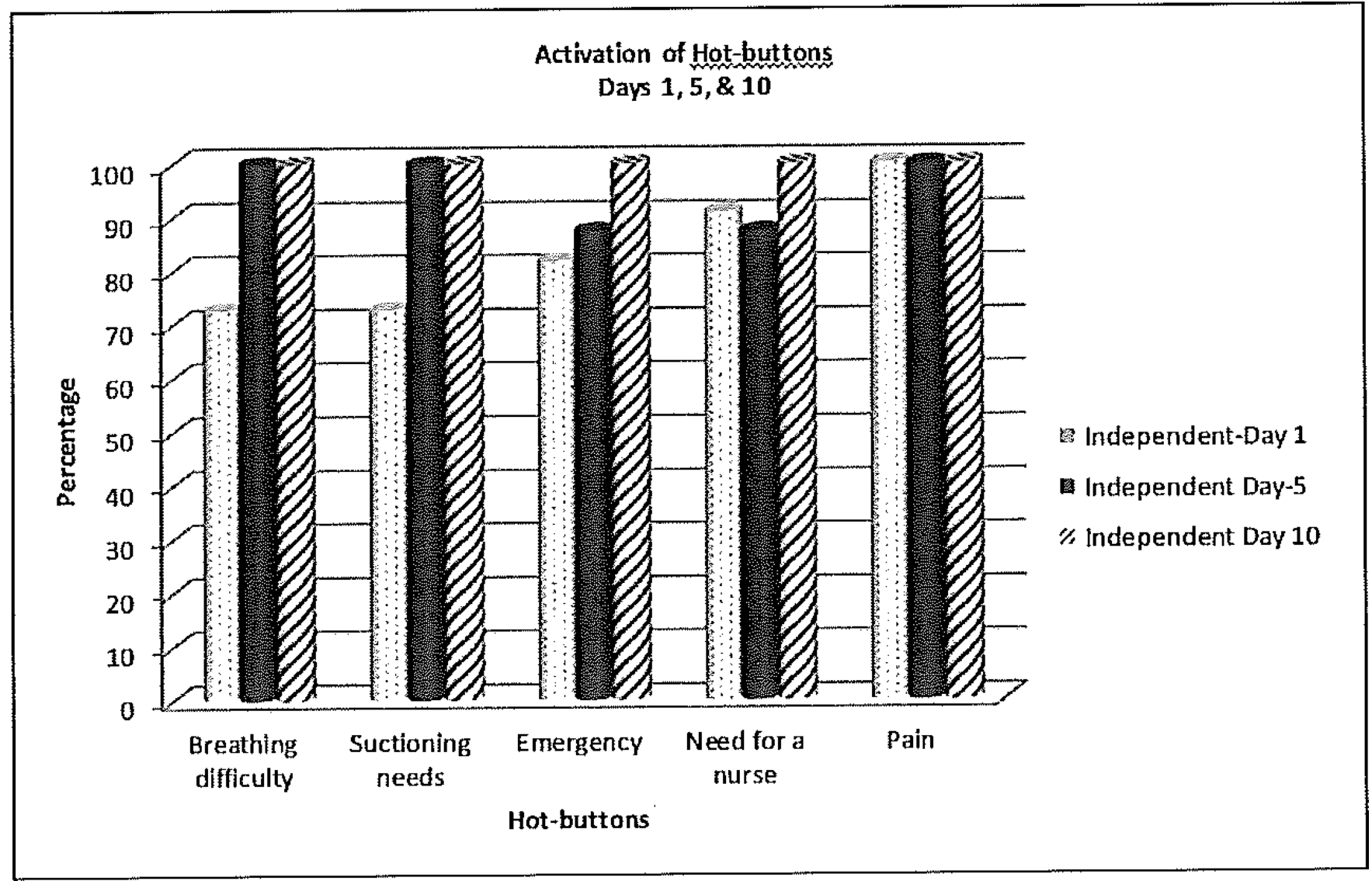

Fig. 1.

Activation of Hot-buttons, Days 1, 5, and 10. 


\section{Table 1}

\section{Study instruments}

\begin{tabular}{|c|c|c|c|}
\hline Variables & & Instrument & Timeline \\
\hline$\bullet$ & Demographic data & $\begin{array}{l}\text { The Demographic \& Clinical Survey: used to collect data about age, } \\
\text { gender, reason for SS, ethnicity, years of education, previous experience } \\
\text { with SS, and support obtained while experiencing SS and hospitalized. }\end{array}$ & $\begin{array}{l}\text { Upon initiation of } \\
\text { study. }\end{array}$ \\
\hline$\bullet$ & $\begin{array}{l}\text { Presence of delirium, } \\
\text { agitation/sedation }\end{array}$ & $\begin{array}{l}\text { The Confusion Assessment Method (CAM) and the Richmond Assessment } \\
\text { Scale (RAS) have established reliability to assess for the presence of } \\
\text { delirium and/or agitation/sedation }[14,15] \text {. }\end{array}$ & $\begin{array}{l}\text { Pre-screening prior to } \\
\text { consent process and } \\
\text { daily completion of } \\
\text { measures. }\end{array}$ \\
\hline - & $\begin{array}{l}\text { Ability to use SGD on } \\
\text { command } \\
\text { Difficulties encountered }\end{array}$ & $\begin{array}{l}\text { The Usability of Communication Intervention Form: used to evaluate } \\
\text { participants' ability to use the SGD system, difficulties encountered, } \\
\text { technology functionality, and clerk's understanding of message via unit } \\
\text { call button. Participants' ability to use device on command is evaluated } \\
\text { with the following coding: } 1=\text { independent; } 2=\text { minimal assistance }(2 \\
\text { prompts or less); } 3=\text { needs considerable assistance }(>2 \text { prompts); } 4= \\
\text { unable to perform). Successfully used by SS patients during previous pilot } \\
\text { study [12]. }\end{array}$ & $\begin{array}{l}\text { Daily for } 10 \text { days or } \\
\text { less based on } \\
\text { participants' discharge. }\end{array}$ \\
\hline$\bullet$ & $\begin{array}{l}\text { Level of importance and } \\
\text { satisfaction with the use of } \\
\text { the SGD }\end{array}$ & $\begin{array}{l}\text { The Patient Satisfaction and Usability Instrument measured the } \\
\text { satisfaction (5 items) and importance level ( } 4 \text { items) associated with the } \\
\text { use of the SGD. Item scores range from } 1 \text { to } 5 \text { with } 1 \text { indicating greater } \\
\text { satisfaction with the item. Successfully used by SS patients on previous } \\
\text { pilot study [12]. Adapted from Demers }[16,17] .\end{array}$ & $\begin{array}{l}\text { Twice before discharge } \\
\text { (to collect reliability } \\
\text { data) or day } 10 .\end{array}$ \\
\hline
\end{tabular}




\section{Table 2}

Patient demographics and clinical characteristics

\begin{tabular}{lc}
\hline Variable & Frequency $(\%)$ \\
\hline Age & $3(27.3 \%)$ \\
$40-50$ years & $4(36.4 \%)$ \\
$51-60$ years & $3(27.3 \%)$ \\
$61-70$ years & $1(9.0 \%)$ \\
$71-80$ years & \\
Gender & $9(81.8 \%)$ \\
Male & $2(18.2 \%)$ \\
Female & \\
Race & $10(90.9 \%)$ \\
White & $1(9.1 \%)$ \\
Black & \\
Years of education & $2(18.2 \%)$ \\
$8-10$ years & $3(27.3 \%)$ \\
$11-13$ years & $5(45.5 \%)$ \\
$14-16$ years & $1(9.0 \%)$ \\
$17-20$ years & $3(27.3 \%)$ \\
Diagnosis leading to SS & $8(72.7 \%)$ \\
Respiratory failure & \\
H and N cancer surgery & \\
\hline
\end{tabular}




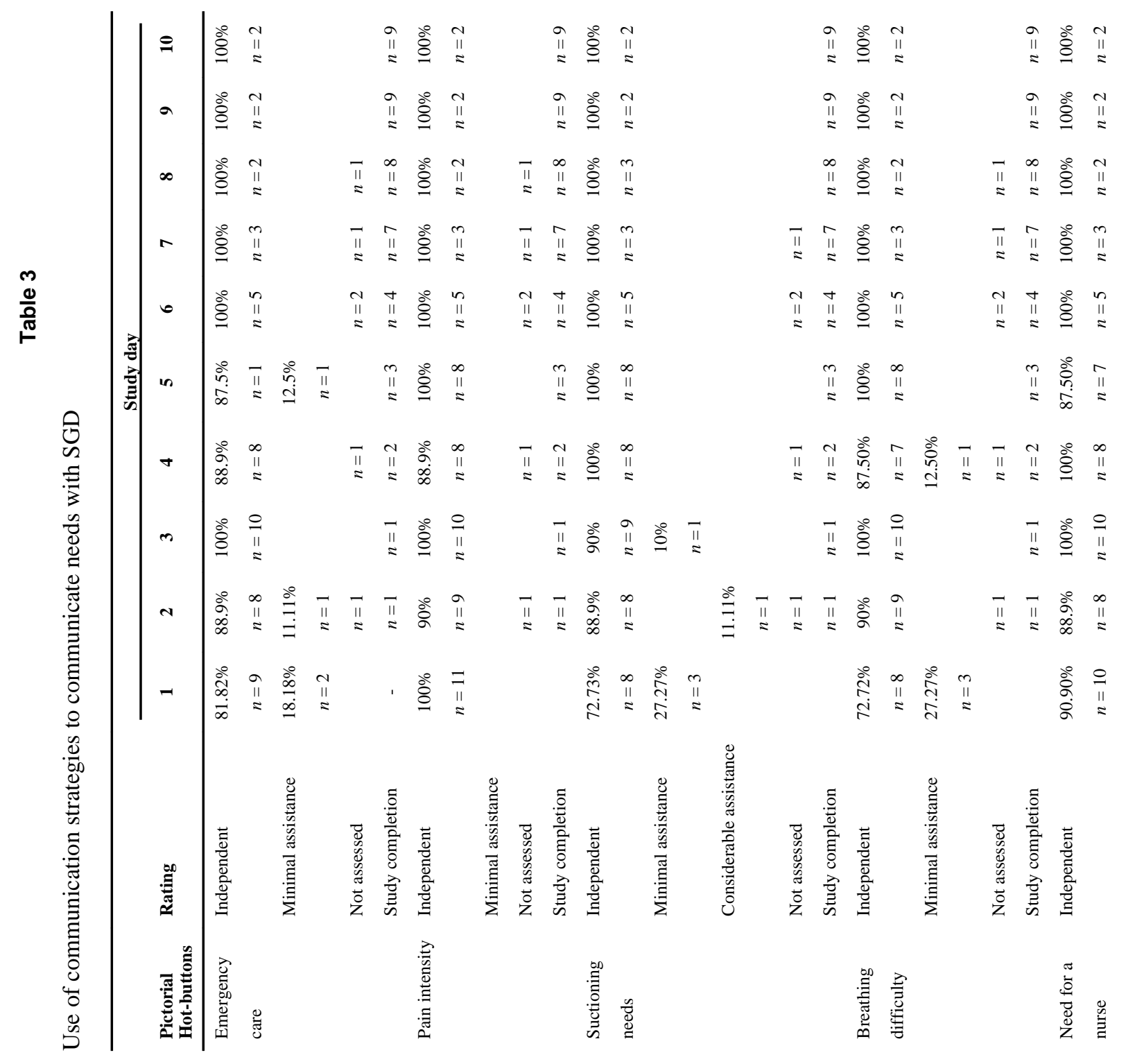




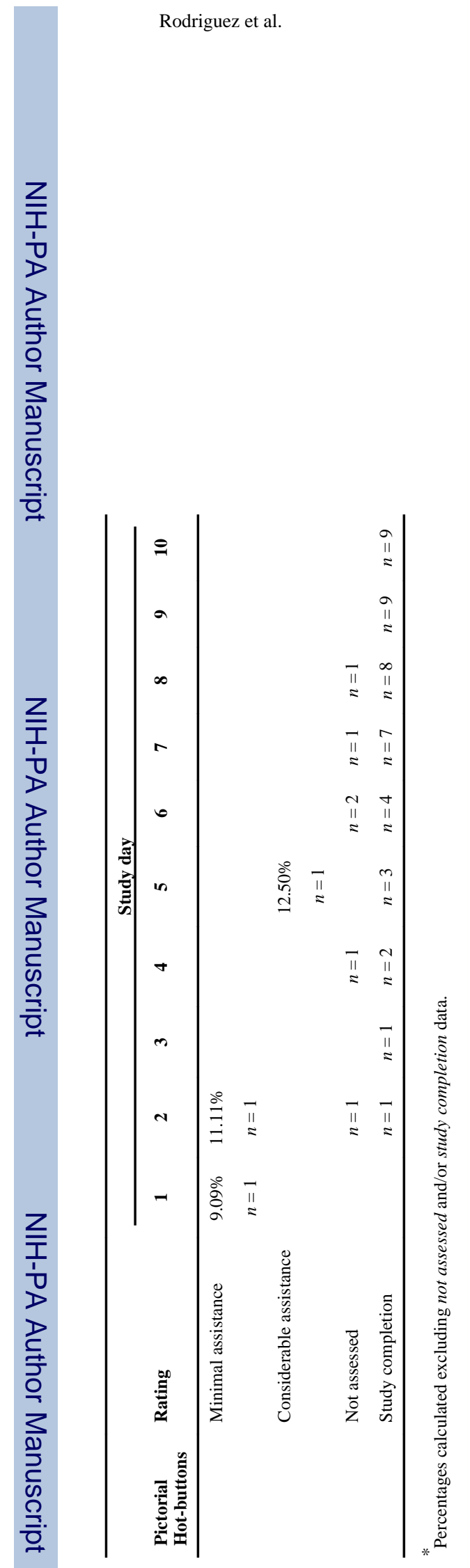

Page 17 